\title{
COMPUTED TOMOGRAPHY (CT) IN DIAGNOSIS AND TREATMENT OF COMPLEX CERVICAL DYSTONIA WITH SEVERE CHRONIC PAIN WITH BOTULINUM TOXIN TYPE A (BTA)
}

\author{
T. Mokina, Y. Pavlov.
}

Pelvic pain and botulinum toxin treatment center, City clinical hospital №29, Moscow, Russia tv mokina@mail.ru, https://www.instagram.com/doctor_mozg

The aim of this case study investigated the use of CT for diagnosis and guidance of BTA treatment in 53-year old male with complex cervical dystonia (left-sided torticollis, torticaput, laterocaput) with horizontal tremor and severe chronic pain.

Methods. The patient received 7 treatments with increasing doses of BTA injected into the superficial neck muscles using standard protocols without CT guidance. To improve outcomes, CT guidance was used during further treatment.

Results. Initial BTA treatments without CT reduced head tremor, but not pain and cervical dystonia (CD).
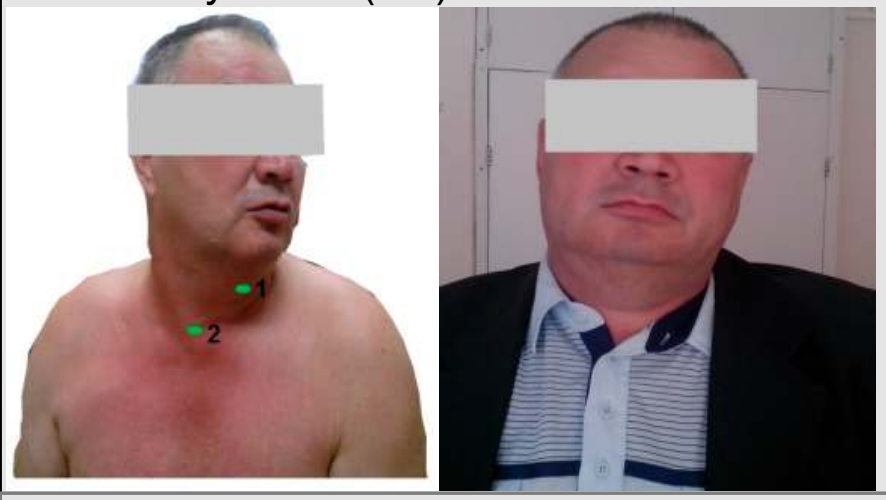

Pic.1. Patient before the BTA treatment, after CTguided injection into the left obliquus capitis inferior he regained the ability to rotate head to the right temporarily. After 2 weeks, he was able to turn his head to the right and keep it in midline; decrease in pain and head tremor was also observed. After 9 CT-guided BTA treatments complete regression of symptoms was observed.

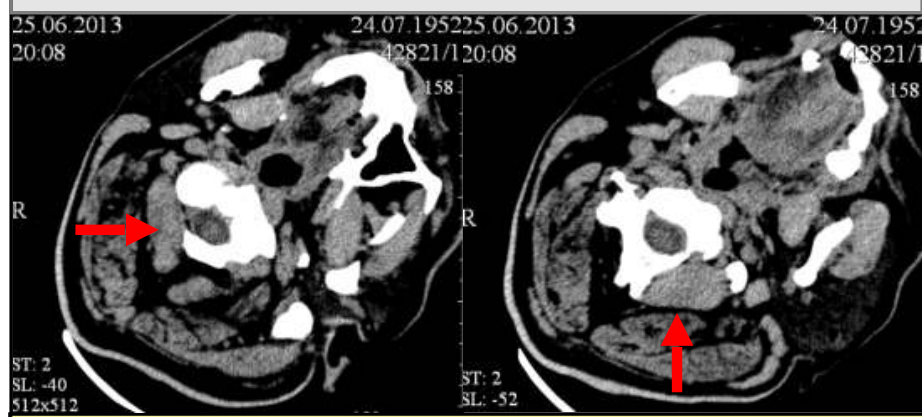

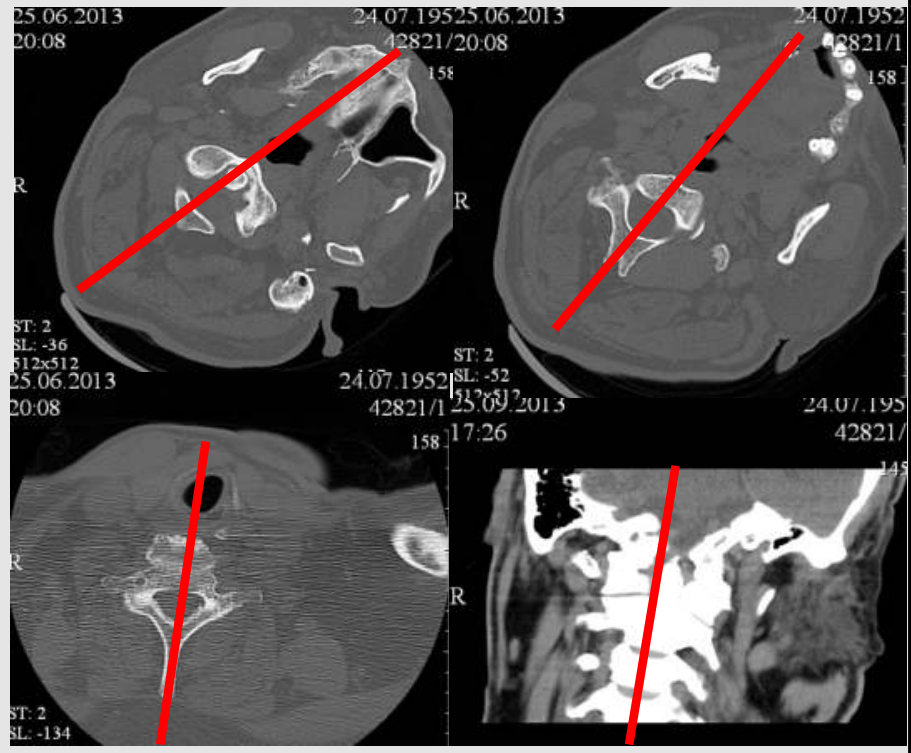

Pic.2. Subsequent CT scans revealed combination of left-sided torticollis, torticaput and laterocaput.

Pic.3. Obliquus capitis inferior muscle $\mathrm{D} 17 \mathrm{~mm}$, $\mathrm{V} 18.5 \mathrm{~cm} 3$ on healthy right side (left pic) and D $21 \mathrm{~mm}, \mathrm{~V} 19.8 \mathrm{~cm} 3$ on the dystonic left side (right pic). Difference $4 \mathrm{~mm}$ and $1.3 \mathrm{~cm} 3$, respectively After 9 CT-guided BTA treatments diameter of dystonic muscle was reduced to the size of the healthy muscle.

Conclusion. CT scanning helped determining aetiology and clinical form of CD, selecting target muscles, guiding BTA injections in deep neck muscles in patients with CD. CT-guidance of BTA injections improved clinical outcome, reduced structural deficiency and chronic pain. As complex CD is common, we recommend more frequent use of CT for diagnosis and BTA treatment.

\section{Bibliography}

1. Bhidayasiri R. Treatment of complex cervical dystonia with botulinum toxin: Involvement of deep-cervical muscles may contribute to suboptimal responses. Parkinsonism Relat Disord. 2011 Nov;17 Suppl 1:S20-4. doi: 10.1016/j. parkreldis.2011.06.015.

2. Reichel G, Stenner A, Jahn A. The phenomenology of cervical dystonia. Fortschr Neurol Psychiatr. 2009 May;77(5):272-7. doi: 10.1055/ s-0028-1109416. Epub 2009 May 5 